\title{
DUST FORMATION IN STELLAR PHOTOSPHERES
}

\author{
The Case of Carbon Stars from Dwarfs to AGB Stars
}

\author{
TAKASHI TSUJI \\ Institute of Astronomy, The University of Tokyo \\ Mitaka, Tokyo, 181 Japan
}

\begin{abstract}
We examine whether dust forms in the photospheres of carbonrich stars by referring to the case of red and brown dwarfs for which some observational clues on dust formation are now known. Dust may form in the photospheres of dwarf carbon stars and produce significant effects on both their structure and spectra. In carbon-rich asymptotic giant branch stars, dust probably forms in the photosphere, if not in the circumstellar envelope, and radiation pressure on dust is sufficient to expel the matter directly from the photosphere. This fact may play some role in mass-loss from cool luminous stars in general, including non-pulsating stars for which no successful mechanism of mass-loss was known.
\end{abstract}

\section{Introduction}

The idea that dust may form in stellar photospheres is not necessarily new, but it has not been known how dust forms in photospheres. For this reason, few attempts have yet been made to include dust in modeling the stellar photosphere. It is only recently that some observational evidence of dust in the photosphere has been recognized in very low-mass stars (VLMSs) and in brown dwarfs. These observations revealed that dust formed in the photospheres of VLMSs may survive as long as the lifetime of these longlived stars, while the dust formed in brown dwarfs may follow a different fate (Tsuji et al. 1996a,b). These observations provide important insight on how dust forms and evolves in the stellar photosphere (Sect. 2).

An interesting case of dust formation in photospheres may be that of the dwarf carbon $(\mathrm{dC})$ stars, the discovery of which is undoubtedly having an important impact in our understanding of the carbon star phenomenon, especially if the space density of $\mathrm{dC}$ stars is much larger than that of carbonrich asymptotic giant branch (AGB) stars (Green, this volume). The dC star may have been formed by binary mass-transfer from an AGB primary, 
and it has preserved a direct probe into the evolution of intermediate mass stars to the AGB stage in the metal-poor era. The large space density of dC stars implies that their progenitors should have contributed significantly to the Galactic chemical evolution, especially of such key elements as C, N, and heavy elements whose origins are by no means well understood yet (Gustafsson, this volume). To clarify the nature of the $\mathrm{dC}$ star, the method of model atmospheres should serve as a useful guide, and dust formation will play an important role especially in view of the rather high condensation temperatures of the dust species expected in the carbon-rich case (Sect. 3 ). In fact, dusty models should be needed for $\mathrm{dC}$ stars with $T_{\text {eff }}<3000 \mathrm{~K}$ even for the low metallicities typical of halo objects (Sect. 4). Although no observation of this is known yet, dust will have appreciable effects on the spectra of cool dC stars. On the other hand, the limited observations now available on relatively warm $\mathrm{dC}$ stars can reasonably be understood by our dust-free models (Sect. 5).

We also found that the necessary condition for dust formation is well met in the photospheres of carbon-rich AGB stars with $T_{\text {eff }}<3000 \mathrm{~K}$. This possibility that dust may form already in the photospheres of AGB stars rather than in the circumstellar envelope has important implications. For example, radiation pressure on dust may provide a long-sought driving force for mass-loss directly from the photospheres of AGB stars. Also, dust formed in photospheres will produce noticeable observable effects (Sect. 6).

\section{An Empirical Approach to Dust Formation in Stellar Photospheres}

The possible presence of dust in stellar photospheres has been shown first for late $\mathrm{M}$ dwarfs, where predicted spectral energy distributions (SEDs) based on models including dust agree rather well with observed ones (Tsuji et al. 1996a). A more clear demonstration of the presence of dust was found in a brown dwarf candidate GD 165B discovered by Becklin \& Zuckerman (1988). In this object, the SED shows evidence of severe extinction by dust, and the molecular bands such as those of $\mathrm{H}_{2} \mathrm{O}$ appear to be too weak to be explained by any model without dust. These observations could be explained first by our dusty models in which dust formed in accordance with the thermodynamical condition of condensation is uniformly mixed with gas throughout (dust-gas homogeneous mixture model). On the other hand, no clear evidence of dust could be seen in the cooler brown dwarf Gl 229B discovered by Nakajima et al. (1995), and its spectrum is dominated by volatile molecules such as methane (Oppenheimer et al. 1995). The observed data for Gl 229B could never be understood by our dusty model but instead can be well explained by our dust-free model (Tsuji et al. 1996b). 
These contrasting observations can be regarded as unique experiments on dust formation in astronomical "laboratories" under different conditions, and they can be interpreted as follows: When the temperature is relatively high as in GD $165 \mathrm{~B}\left(T_{\text {eff }} \approx 1800 \mathrm{~K}\right)$, the dust is still smaller than the critical radius $r_{\mathrm{cr}}$ below which the dust once formed cannot grow and dissolves back to molecules. In this case, small dust grains and gaseous molecules are in detailed balance and hence a certain amount of dust allowed by the thermodynamical equilibrium is always present in the photosphere. This provides a rationale to our simple dust-gas homogeneous mixture model and also a reason why dust can survive as long as the lifetime of VLMSs. On the other hand, if the temperature is relatively low as in Gl $229 \mathrm{~B}$ ( $T_{\text {eff }}$ $\approx 1000 \mathrm{~K}$ ), the dust can be larger than the critical radius $r_{\mathrm{cr}}$ and the dust once formed begins to grow. Then, the detailed balance between dust and gas can no longer be maintained because of the onset of this irreversible process. For proper treatment of such a case, not only the non-equilibrium kinetic theory of dust growth such as has been developed for cool stellar winds (Sedlmayr \& Winters, this volume) but also the methods of meteorology may be needed. Anyhow, dust grows and finally segregates from the dust-gas homogeneous mixture to form a dust aggregation or cloud. Then, volatile molecules can appear through the gaps between dust clouds, as has been observed in Gl 229B, and the dust will no longer be so effective in blocking the emergent radiation as in the dust-gas homogeneous mixture model which applied well to GD 165B.

Now, one important by-product of the recent efforts to clarify the nature of brown dwarfs is a finding that there should be a regime where dust may be formed in LTE and remain in detailed balance with the gas so long as the thermodynamical condition of condensation is fulfilled at the relatively high temperatures of the stellar photosphere. We assume in the following that the photosphere of carbon-rich stars from dwarfs to AGB stars can also be in this dust-gas detailed balance regime, although we will not speak of carbon-rich brown dwarfs for the moment.

\section{Physical Properties of Carbon-Rich Mixture at High Density}

We begin with a simple computation of chemical equilibrium in which condensation is taken into account. An example of a carbon-rich mixture at $\log P_{g}=6.0$ is shown in Figure 1. A noticeable feature in the chemical equilibrium at high density, in contrast to the more familiar case of low density relevant to AGB carbon stars, is the predominance of $\mathrm{CH}_{4}$ instead of CO. This has important consequences: First, $\mathrm{CO}$ can no longer be the major species of carbon. Then, as oxygen in $\mathrm{CO}$ is released, a large amount of $\mathrm{H}_{2} \mathrm{O}$ must be formed. This unexpected but important result that $\mathrm{H}_{2} \mathrm{O}$ 


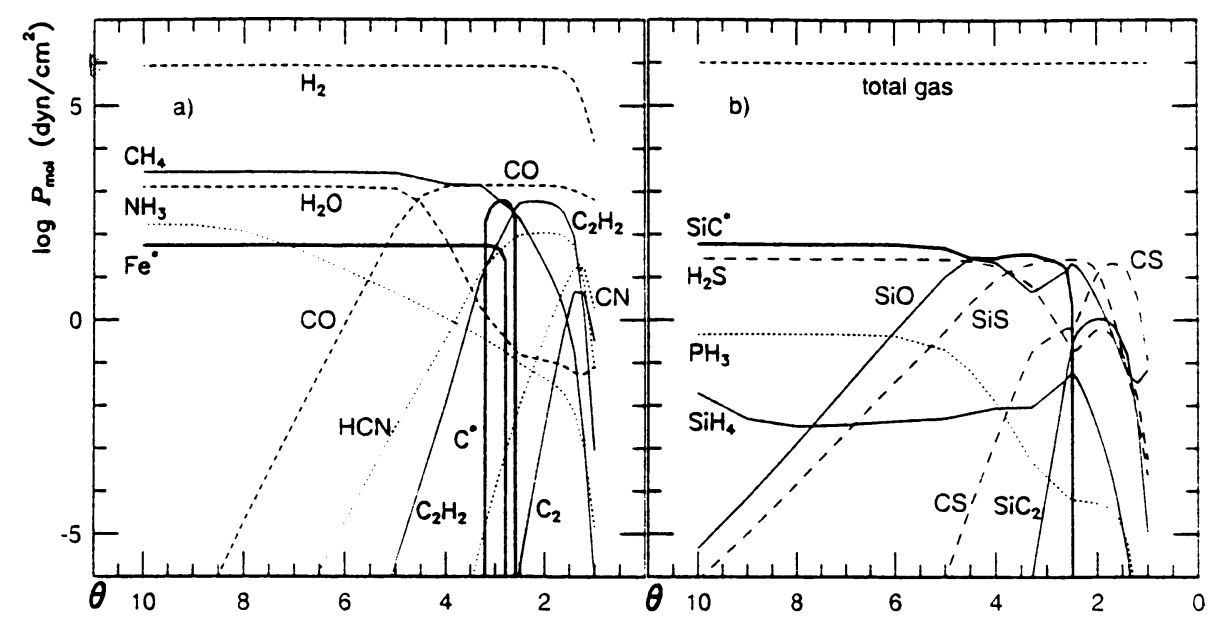

Figure 1. Chemical equilibrium of a carbon-rich mixture $(C$ is increased so that $C / O=2$ in the solar composition mixture) at $\log P_{g}=6.0$. Partial pressures of selected molecules (asterisks indicate fictitious pressures of monomers composed of condensed species) are plotted against $\theta=5040 / T$. (a) Molecules composed of $\mathrm{H}, \mathrm{C}, \mathrm{N}$, and $\mathrm{O}$. Note that $\mathrm{H}_{2} \mathrm{O}$ dominates at $T<1000 \mathrm{~K}$ even for $\mathrm{C}>\mathrm{O}$. (b) Molecules including $\mathrm{S}, \mathrm{Si}$, and $\mathrm{P}$.

dominates even in a carbon-rich mixture at low temperature and high pressure seems to have been overlooked until the present. Thus, a high density, cool atmosphere is dominated by $\mathrm{H}_{2} \mathrm{O}$ as well as by $\mathrm{CH}_{4}$ even if $\mathrm{C}>\mathrm{O}$, and $\mathrm{CO}$ formation no longer plays such a special role as to characterize oxygenrich and carbon-rich atmospheres. Second, although graphite is formed at relatively high temperature (about $2500 \mathrm{~K}$ ), it soon disappears under high pressure (at about $1500 \mathrm{~K}$ under $\log P_{g}=6.0$ ), while graphite can survive to lower temperatures under low pressure. This somewhat unexpected result, however, is not a new one but has been noticed before (Tsuji 1964).

Now, in extending our opacity code for carbon-rich mixtures to high densities, we identify three major problems: First, we must consider the infrared bands of almost all the molecules that appear in Fig. 1, namely, $\mathrm{CO}$, $\mathrm{H}_{2} \mathrm{O}, \mathrm{C}_{2} \mathrm{H}_{2}, \mathrm{CH}_{4}, \mathrm{HCN}, \mathrm{NH}_{3}, \mathrm{SiH}_{4}, \mathrm{SiO}, \mathrm{SiS}, \mathrm{CS}, \mathrm{H}_{2} \mathrm{~S}$, and $\mathrm{PH}_{3}$ together with the electronic bands of $\mathrm{CH}, \mathrm{C}_{2}, \mathrm{CN}, \mathrm{CaH}, \mathrm{MgH}$, and $\mathrm{FeH}$. We treat all these molecular opacities by the band model method throughout (Tsuji 1994). For the reason already mentioned, we should include $\mathrm{H}_{2} \mathrm{O}$ opacity even for $\mathrm{C}>\mathrm{O}$ at high density. Second, abundant $\mathrm{H}_{2}$ is now an important source of opacity at high density because of the collision-induced absorption (CIA), whose absorption coefficients have been updated by Borysow (1994). The importance of $\mathrm{H}_{2}$ CIA relative to other sources of opacity is essentially the same as in the oxygen-rich case (Tsuji \& Ohnaka 1995). Third, we must represent the effects of dust opacities by amorphous carbon (C), silicon 


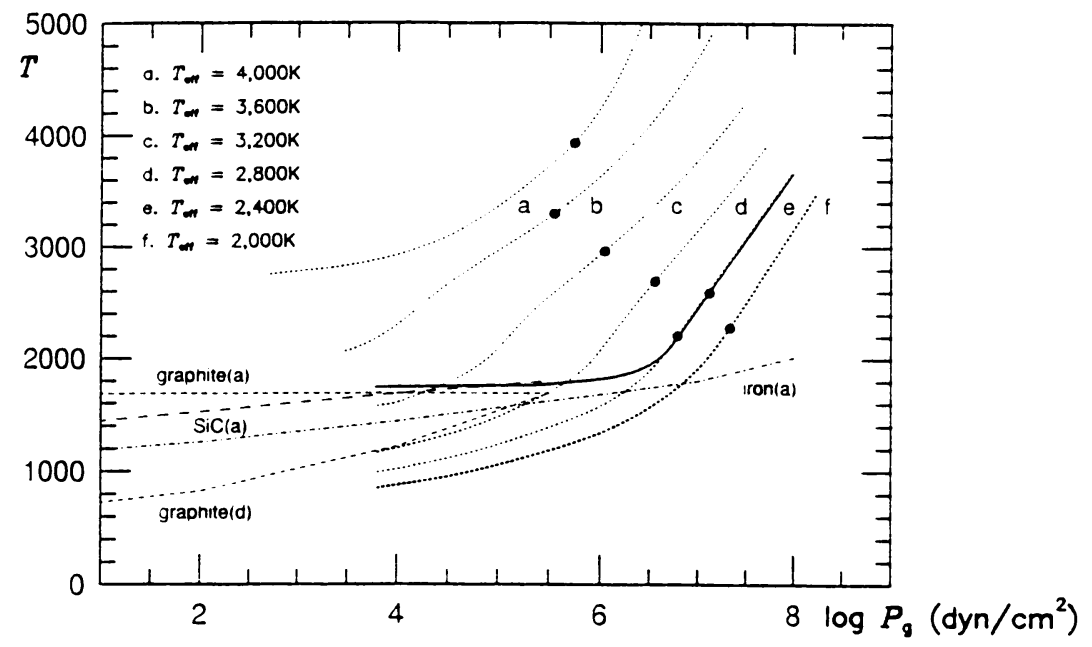

Figure 2. Dust-free model atmospheres of dC stars with $Z / Z_{\odot}=0.1$ and $\mathrm{C} / \mathrm{O}=2$ are shown by dotted lines for $T_{\text {eff }}$ between 2000 and $4000 \mathrm{~K}\left(\log g=5.0\right.$ and $v_{\text {micro }}=1.0 \mathrm{~km}$ $\mathrm{s}^{-1}$ throughout). The appearance-lines for graphite, silicon carbide, and iron, as well as the disappearance-line for graphite, are indicated. An example of a dusty model is shown by the solid line for $T_{\text {eff }}=2400 \mathrm{~K}$. Dusty models of other $T_{\text {eff }}$ show essentially the same structure. Filled circles indicate the onset of convection.

carbide $(\mathrm{SiC})$, and iron $(\mathrm{Fe})$. As we are assuming that the size of the dust species is still smaller than the critical radius $r_{\text {cr }}$ (Sect. 2), the extinction coefficient can be evaluated by a series expansion of the Mie formula (van de Hulst 1957).

\section{Photospheres of Dwarf Carbon Stars with and without Dust}

We first considered gaseous opacities alone and constructed a small grid of model atmospheres for dC stars which covers $T_{\text {eff }}$ between 2000 and $4000 \mathrm{~K}$ with a step of $400 \mathrm{~K}$ ( $\log g=5.0$ and $v_{\text {micro }}=1.0 \mathrm{~km} \mathrm{~s}^{-1}$ throughout). We assumed metallicities of $Z / Z_{\odot}=1,0.1$, and 0.01 in which the relative abundances are solar except for $\mathrm{C} / \mathrm{O}=2(\mathrm{C}$ increased). The resulting models in radiative-convective equilibrium, for the case of $Z / Z_{\odot}=0.1$ as an example, are shown by the dotted lines in Figure 2. The appearance temperatures for graphite, silicon carbide, and iron, as well as the disappearance temperatures for graphite, are plotted against $\log P_{g}$ in Fig. 2. As discussed in Sect. 3, graphite condenses only in a limited regime of the phase diagram. Figure 2 reveals that only the models for $T_{\text {eff }}=4000$ and $3600 \mathrm{~K}$ can be dust-free, while all the models with $T_{\text {eff }}<3200 \mathrm{~K}$ are not self-consistent in that dust formation is neglected, contrary to the thermodynamical result. The results are not much different for other metallicities, although dust tends to appear in models of higher $T_{\text {eff }}$ at higher metallicity. 


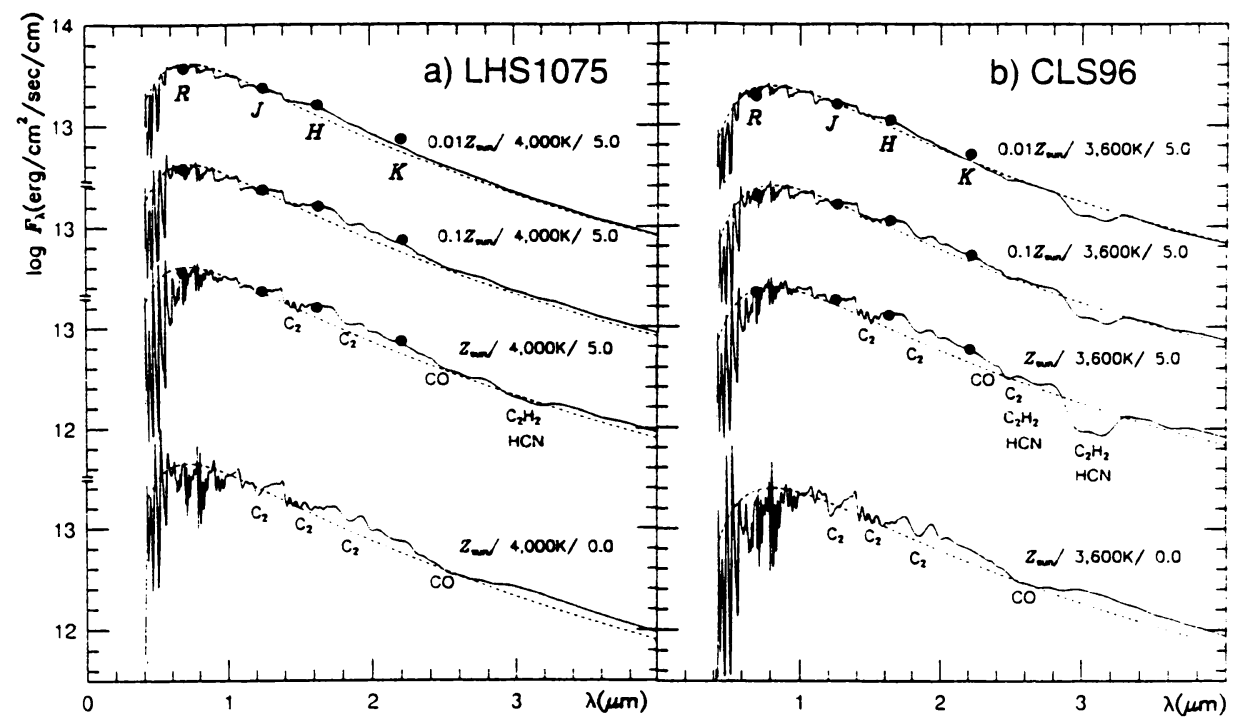

Figure 3. (a) Predicted SEDs based on our models for a dC star with $T_{\text {eff }}=4000 \mathrm{~K}$ are shown by solid lines (dashed lines are blackbody curves for $4000 \mathrm{~K}$ ) for $Z / Z_{\odot}=0.01$, 0.1 , and 1 ( $C / O=2$ throughout), and compared with the observed SED (filled circles) of the dC star LHS 1075 based on $R$ (Green et al. 1991) and JHK (Bothun et al. 1991) photometry. The predicted SED for a carbon-rich AGB star of the same $T_{\text {eff }}$ is also shown at the bottom, and a significant luminosity effect can be seen $\left(Z / T_{\text {eff }} / \log g\right.$ is indicated for each SED). (b) The same for the case of $T_{\text {eff }}=3600 \mathrm{~K}$ and the dC star CLS 96.

Now, dust should form in the photospheres of dC stars with $T_{\text {eff }}<$ $3200 \mathrm{~K}$. Modeling a dusty photosphere, however, was more difficult in the carbon-rich case than in the oxygen-rich case. In fact, the heating by highly absorbing forms of dust such as graphite is so large that the dust appears only at the very surface of the photosphere, in marked contrast to the oxygen-rich case where dust could form deeper in the photosphere (Tsuji et al. 1996a). The resulting dusty model for a dC star of $T_{\text {eff }}=2400 \mathrm{~K}$ in radiative-convective equilibrium is shown by the solid line in Fig. 2. A small amount of dust condensing at the very surface gives a drastic heating of nearly $1000 \mathrm{~K}$.

\section{Some Observable Properties of Dwarf Carbon Stars}

The predicted SEDs based on our models for $T_{\text {eff }}=4000$ and $3600 \mathrm{~K}$ for the cases of $Z / Z_{\odot}=0.01,0.1$, and 1 are shown in Figure 3 by the solid lines. Compared with the SED based on a model of an AGB carbon star of the same $T_{\text {eff }}$ shown at the bottom of each panel, molecular bands such as those of $\mathrm{CO}(2.3 \mu \mathrm{m})$ are much weaker in the model for $\mathrm{dC}$ stars. This prediction agrees nicely with recent observations of the infrared spectra of $\mathrm{dC}$ stars 


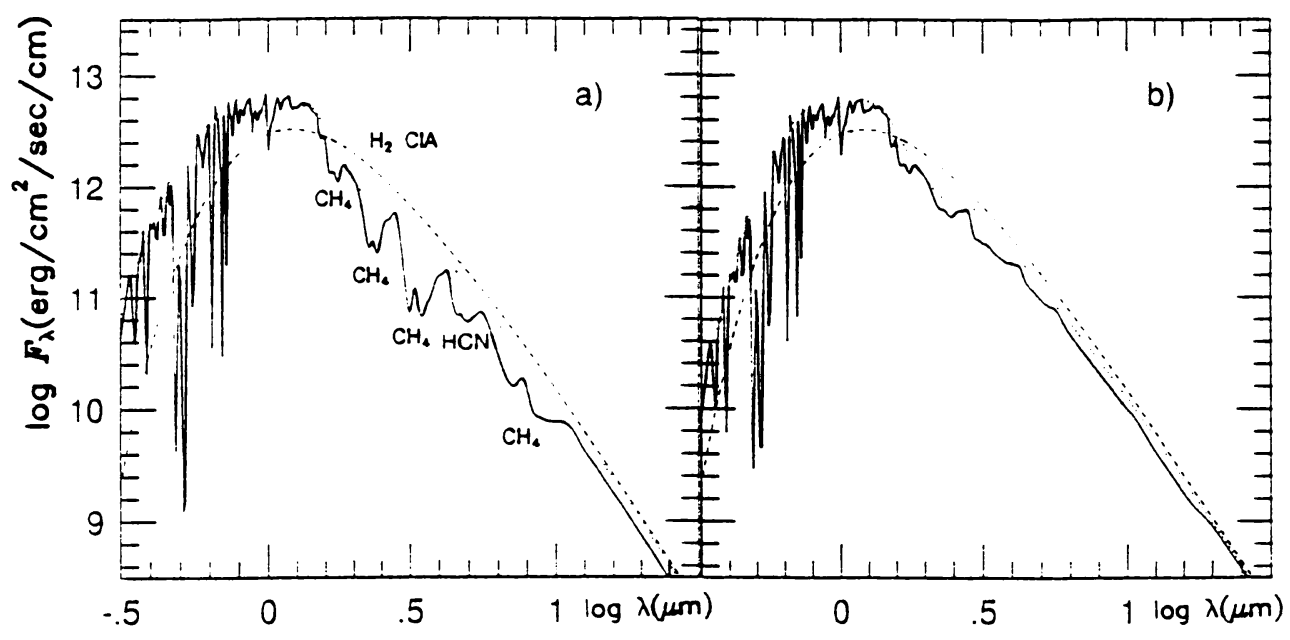

Figure 4. Predicted SEDs for (a) dust-free and (b) dusty models of a dC star with $T_{\text {eff }}=2400 \mathrm{~K}\left(Z / Z_{\odot}=0.1, \mathrm{C} / \mathrm{O}=2, \log g=5.0\right.$, and $\left.v_{\text {micro }}=1.0 \mathrm{~km} \mathrm{~s}^{-1}\right)$ are shown by the solid lines. The dotted and dashed lines are the emergent fluxes for continuous opacities alone (including $\mathrm{H}_{2}$ CIA; dust opacities are also included in the dusty case) and blackbody curves, respectively.

(Joyce, this volume). On the other hand, absorption bands due to HCN and $\mathrm{C}_{2} \mathrm{H}_{2}(3.1 \mu \mathrm{m})$ appear already at $T_{\text {eff }}=4000 \mathrm{~K}\left(Z=Z_{\odot}\right)$ and are much stronger at $T_{\text {eff }}=3600 \mathrm{~K}$ (even with low $Z$ ) in $\mathrm{dC}$ star models, while they are invisible in the AGB models of $T_{\text {eff }}=4000$ and $3600 \mathrm{~K}\left(Z=Z_{\odot}\right)$. Thus the infrared spectra show significant luminosity effects in carbon-rich stars. The observed SEDs of the dC stars LHS 1075 and CLS 96 (filled circles) are compared with the predicted ones in Figs. $3 a$ and $3 b$, respectively. It appears that $T_{\text {eff }}$ may be close to $4000 \mathrm{~K}$ for LHS 1075 and close to $3600 \mathrm{~K}$ for CLS 96. In both $\mathrm{dC}$ stars, the case of $Z=0.1 Z_{\odot}$ provides the best overall fit. It may still be premature to suggest that the metallicity can be estimated this way, especially because of the possibility that the relative abundances of the elements may be highly non-solar in $\mathrm{dC}$ stars.

As an example of cooler dC stars, the SED predicted by the dust-free model for $Z=0.1 Z_{\odot}$ and $T_{\text {eff }}=2400 \mathrm{~K}$ is shown in Figure $4 a$ by the solid line. Now, the infrared region $(\lambda>1.5 \mu \mathrm{m})$ shows a severe depression by strong molecular bands, mostly of $\mathrm{CH}_{4}$ (note that $\mathrm{HCN}$ and $\mathrm{C}_{2} \mathrm{H}_{2}$ are already rather weak). Further, quasi-continuous $\mathrm{H}_{2}$ CIA plays an important role and this effect is more important in more metal-poor cases. As a result of the large opacities at $\lambda>1.5 \mu \mathrm{m}$, the SED shows a large excess shortward of $1.5 \mu \mathrm{m}$ and peaks at about $1 \mu \mathrm{m}$. Because of the increasing importance of $\mathrm{CH}_{4}$ and $\mathrm{H}_{2} \mathrm{CIA}$ at the lower luminosity, the spectra again show significant luminosity effects (compare Figures 4 and 6 with the difference in 


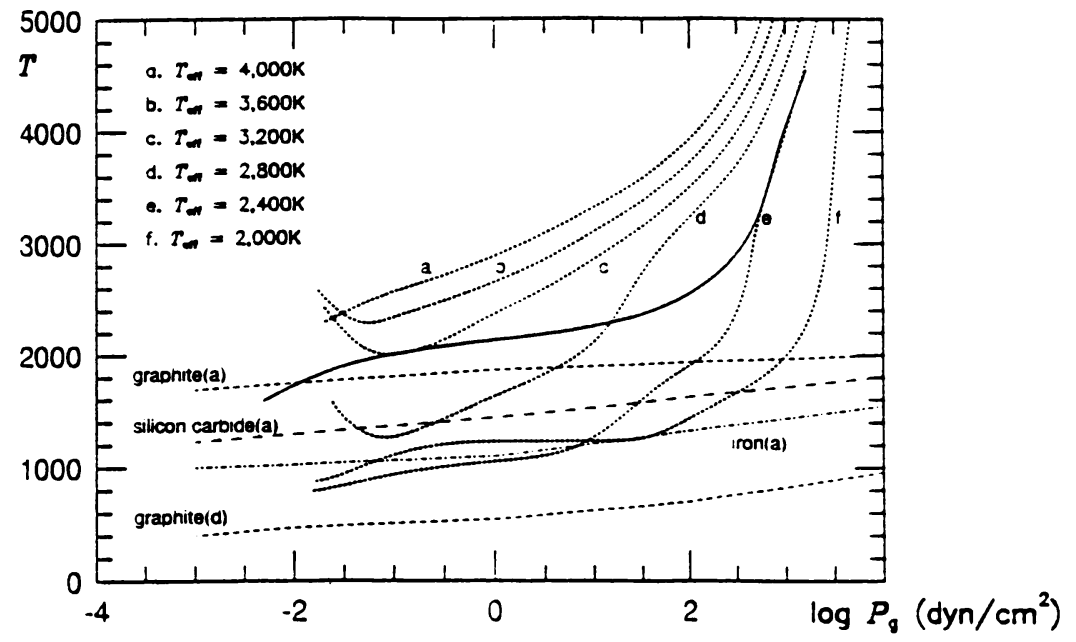

Figure 5. Dust-free model atmospheres of carbon-rich AGB stars with $Z / Z_{\odot}=1.0$ and $\mathrm{C} / \mathrm{O}=2$ are shown by dotted lines for $T_{\text {eff }}$ between 2000 and $4000 \mathrm{~K}(\log g=0.0$ and $v_{\text {micro }}=3.0 \mathrm{~km} \mathrm{~s}^{-1}$ throughout). An example of a dusty model in radiative equilibrium is shown by the solid line for $T_{\text {eff }}=2400 \mathrm{~K}$. Dusty models of other $T_{\text {eff }}$ show essentially the same structure. As to other details, see the legend to Fig. 2.

metallicity in mind), but the effects are somewhat different from those in the hotter case noted above. If dust formation is introduced in this model, the SED shows a drastic change as shown by the solid line in Fig. $4 b$. In particular, molecular bands are now much weaker, reflecting the higher photospheric temperature. However, the SED still shows a large excess in the $1 \mu \mathrm{m}$ region. At present, no observation of such a cool dC star is known, but we hope that our prediction can be of some use as a guide for future searches for such objects.

\section{Dust Formation in Photospheres of Carbon-Rich AGB Stars}

The belief that high density is more favorable for dust formation is not necessarily true, at least for graphite (Sect. 3). In fact, the thermodynamical condition for condensation is well fulfilled for cool AGB stars (solar abundance except for $\mathrm{C} / \mathrm{O}=2, \log g=0.0$, and $v_{\text {micro }}=3.0 \mathrm{~km} \mathrm{~s}^{-1}$ ) with $T_{\text {eff }}<3000 \mathrm{~K}$, as shown in Figure 5 , and dust-free models can no longer be self-consistent for them. It is possible to recover the thermodynamical equilibrium by including dust formation in our modeling, and an example of a dusty model in radiative equilibrium is shown by the solid line in Fig. 5 for $T_{\text {eff }}=2400 \mathrm{~K}$. Again, a small amount of dust formed at the very surface produces an enormous effect on the thermal structure of the photosphere.

What is more interesting, the effective gravity turns out to be negative $\left(g_{\text {eff }}=g-g_{\mathrm{rad}}<0\right)$ due to radiation pressure on the dust in the dusty model 


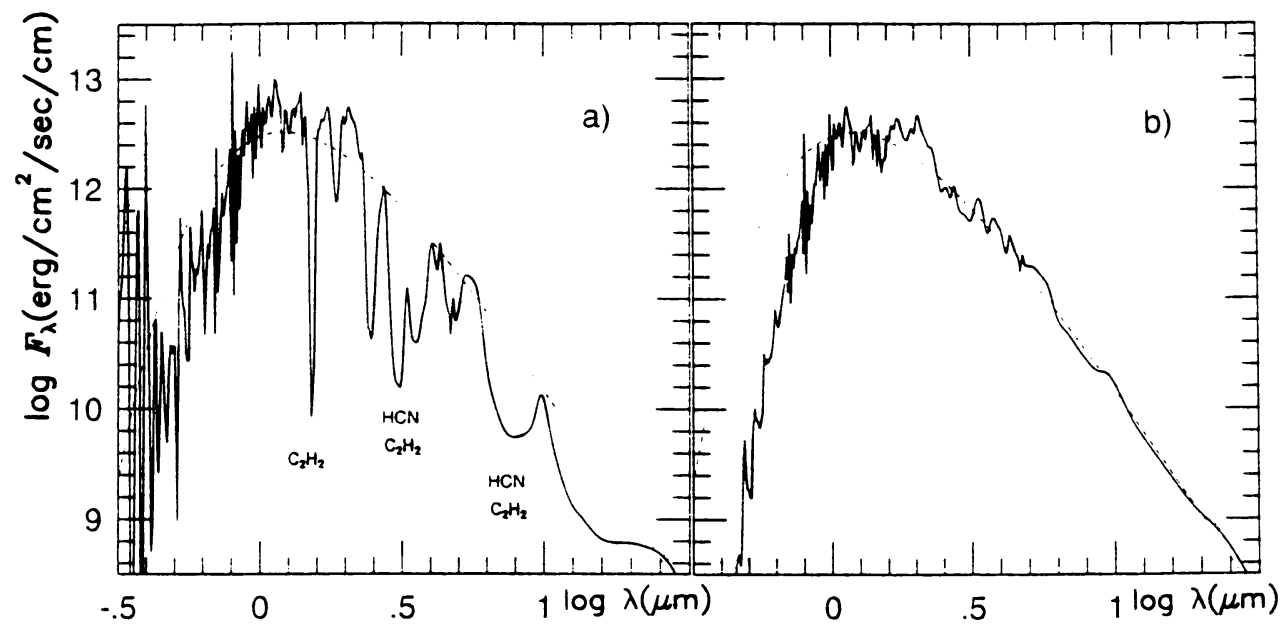

Figure 6. Predicted SEDs for (a) dust-free and $(b)$ dusty models of a carbon-rich AGB star with $T_{\text {eff }}=2400 \mathrm{~K}\left(Z / Z_{\odot}=1.0, \mathrm{C} / \mathrm{O}=2, \log g=0.0\right.$, and $\left.v_{\text {micro }}=3.0 \mathrm{~km} \mathrm{~s}^{-1}\right)$ are shown by the solid lines. As to other details, see the legend to Fig. 4.

photosphere of AGB stars. This fact implies that a stable photosphere can no longer be possible and that a mass-loss outflow initiates directly from the photosphere. It is now generally believed that radiation pressure on dust is the major driving force for mass-loss from cool evolved stars. However, dust is generally assumed to be formed in the circumstellar envelope where the temperature may be below the condensation temperature but the density may be too low for dust to form. This difficulty has been solved for the case of Mira variables by levitating the mater to the condensation point by pulsation (Fleischer et al., this volume). So far, however, no mechanism of mass-loss for general cases other than the pulsating Miras is known, since dust can no longer be formed in the circumstellar envelope.

We now find that the surface temperatures of carbon stars with $T_{\text {eff }}$ $<3000 \mathrm{~K}$ can be very low ( 1000 K, see Fig. 5 ) due to their highly non-gray molecular opacities, and dust can easily be formed in the photosphere. Actually, the formation of dust results in a strong heating of the photosphere, but a small amount of dust can always be formed in radiative equilibrium, since otherwise the temperature drops again drastically. Then the outward acceleration due to radiation pressure on the dust is sufficient to overcome the stellar surface gravity in AGB stars, and an outflow originating directly from the photosphere can provide a simple yet physically clear mechanism for stellar mass-loss from cool evolved stars, for general cases other than the pulsating Miras. Previously, radiation pressure on molecules had been considered as an outward driving force, but it appeared not to be sufficient 
for stellar mass-loss either in O-rich (Tsuji 1978) or in C-rich (Jørgensen \& Johnson 1992) cases. Now, dust can do easily what molecules could not.

In Figure $6 a$, the SED predicted by a dust-free model for $Z=Z_{\odot}, \mathrm{C} / \mathrm{O}$ $=2$, and $T_{\text {eff }}=2400 \mathrm{~K}$ is shown by the solid line. A noticeable feature here is the enormously deep absorption due to $\mathrm{HCN}$ and $\mathrm{C}_{2} \mathrm{H}_{2}$. However, no observation of such strong absorption is known in real carbon stars. If dust formation is introduced in this model, the SED shows a drastic change as shown by the solid line in Fig. $6 b$. The molecular bands are now much weaker, reflecting the higher photospheric temperatures and extinction by the dust. Although we have not yet incorporated the dynamics of mass outflow, a small amount of dust formed in the photosphere will give more or less similar observable effects. Such an SED can be more compatible with the observations so far known, and this can be taken as observational evidence that dust forms already in the photosphere. We hope that this problem can be clarified by future observations, especially by ISO.

\section{Concluding Remarks}

We have investigated the characteristic features of dust formation in the photospheres of carbon-rich stars, where thermodynamics indicates that dust condenses in a wide range of physical conditions. However, once the constraint of radiative equilibrium is introduced, the temperature of the photosphere rises enormously and only a small amount of dust appears at the very surface of the photosphere. This is fatal for the absorbing dust species expected in the carbon-rich case, in contrast to the non-absorbing silicates in the oxygen-rich case. Nevertheless this small amount of dust, if formed in the photosphere, will have significant effects on both the photospheric structure and the spectra, not only of dwarf carbon stars but also of AGB stars. In AGB stars, however, hydrodynamics must be introduced already in the photosphere because of the negative effective gravity due to radiation pressure on the dust. This in turn opens the interesting possibility that a radiatively-driven wind can be realized in cool evolved stars other than the Mira variables, since dust can be formed in the photosphere even if not formed in the circumstellar envelope. Whether this can explain the large mass-loss observed in cool luminous stars in general remains to be examined further.

\section{References}

Becklin, E. E. \& Zuckerman, B. 1988, Nature, 336, 656

Borysow, A. 1994, in Molecules in the Stellar Environment, ed. U. G. Jørgensen (Springer), p. 209

Bothun, G., Elias, J.H., MacAlpine, G., Matthews, K., Mould, J.R., Neugebauer, G. \& Reid, I. N. 1991, $A J, 101,2220$ 
Green, P. J., Morgan, B. \& MacConnell, D. J. 1991, ApJ, 380, L31

Jørgensen, U. G. \& Johnson, H. R. 1992, $A \& A, 265,168$

Nakajima, T., Oppenheimer, B. R., Kulkarni, S. R., Golimowski, D. A., Matthews, K. \& Durrance, S. T. 1995, Nature, 378, 464

Oppenheimer, B.R., Kulkarni, S.R., Matthews, K. \& Nakajima, T. 1995, Science, 270, 1478

Tsuji, T. 1964, Ann. Tokyo Astron. Obs., 2nd Ser., 9, 1

Tsuji, T. $1978, A छ A, 62,29$

Tsuji, 'T. 1994, in Molecules in the Stellar Environment, ed. U. G. Jørgensen (Springer), p. 79

Tsuji, T. \& Ohnaka, K. 1995, ASP Conf. Series, 78, 69

Tsuji, T., Ohnaka, K. \& Aoki, W. 1996a, $A \& A$, 305, L1

Tsuji, T., Ohnaka, K. Aoki, W. \& Nakajima, T. 1996b, $A \& A$, 308, L29

van de Hulst, H. C. 1957, Light Scattering by Small Particles (J. Wiley \& Sons)

\section{Discussion}

Dorfi: In the case of dust formation in AGB atmospheres, a massive stellar outflow will develop which modifies the density and temperature structure of the outer atmosphere. How can you calculate spectra of these models assuming an atmosphere in hydrostatic equilibrium?

Tsuji: As I mentioned, the effective gravity becomes negative $\left(g_{\mathrm{eff}}<0\right)$ if dust forms in an AGB photosphere, and the atmosphere is no longer in hydrostatic equilibrium. In our very preliminary test model of a dusty AGB photosphere, we switched off the radiation pressure term and artificially recovered hydrostatic equilibrium. The reason why I showed this very preliminary spectral energy distribution for a dusty AGB photosphere was just to illustrate qualitatively the possible enormous effects of a small amount of dust forming in the photosphere (not in the circumstellar envelope), especially because this may relax one of the major difficulties in our interpretation of the observed spectral energy distributions of very cool carbon stars. A more self-consistent treatment is certainly needed to see the full consequences of dust formation in the photospheres of AGB stars, both on their spectral energy distributions and on mass outflows.

[Note added in proof: After the meeting, we examined the dynamical effect by keeping the radiation pressure term in the momentum equation. Because of the dust formed in the photosphere, the sonic point is found within the photosphere, and it turns out that the wind velocity is several hundred $\mathrm{km} \mathrm{s}^{-1}$ at the stellar surface but the mass-loss rate is only of the order of $10^{-10} M_{\odot}$ per year. From this result, we think it more difficult for the massive wind to originate directly from the photosphere even if dust forms in the photosphere. On the other hand, a less massive but high-speed wind may be possible. Such a wind may not necessarily be directly observable 
but may have some effects on both the atmospheric structure and mass-loss of AGB carbon stars.]

Lloyd Evans: A possibly relevant observation is that, in the dwarf carbon star LHS 1075 , though the $J H K$ colors and spectrum at $5000 \AA$ indicate it is a warm carbon star like the $\mathrm{CH}$ stars in $\omega$ Cen, at $4000 \AA$ it has the weak $\mathrm{CN}$ bands and $\mathrm{Ca}$ II $\mathrm{H} \& \mathrm{~K}$ lines of the coolest giant carbon stars (Swings-Struve effect). But this might result instead from a low abundance of elements such as $\mathrm{Ca}$ and $\mathrm{N}$.

Tsuji: It is very interesting to hear some details about the spectra of dwarf carbon stars. As for the $\mathrm{CN}$ bands and $\mathrm{Ca}$ II $\mathrm{H} \& \mathrm{~K}$ lines, these may show a significant luminosity effect as can be inferred from the oxygen-rich case, but I have not examined this yet. I also agree with you that the metallicity plays some role, and the possibility that the relative abundances are highly non-solar makes the problem more complicated. As to the violet spectra of very cool carbon stars, the so-called UV depression in these stars is not yet fully understood, and I suppose that extinction by the dust that may form in the photospheres of very cool carbon stars may play some role. 